\title{
Squamous Cell Carcinoma Manifested as a Cutaneous Horn: A Key to Early Detection
}

\author{
Ryoko Kimura, Kazunari Sugita, Hiroyuki Goto and Osamu Yamamoto \\ Division of Dermatology, Department of Medicine of Sensory and Motor Organs, School of Medicine, Tottori University Faculty of \\ Medicine, Yonago 683-8504, Japan
}

\begin{abstract}
We report a case of squamous cell carcinoma manifested as a cutaneous horn. A 92-year-old man was referred to our department for evaluation of a 3-month history of a keratotic cutaneous horn on the left side of his neck. The height of the cutaneous horn was larger than the diameter of erythema at the base. Histopathological examination showed a hyperkeratotic horn developing over a mass of large atypical keratinocytes with large hyperchromatic and pleomorphic nuclei. Based on these findings, a diagnosis of squamous cell carcinoma was made. A recent study showed that cutaneous horns in association with invasive squamous cell carcinoma had a tendency to have less height than the diameter of the base. In our case, however, the height of the cutaneous horn was larger than the diameter of the base, indicating that invasive squamous cell carcinoma sometimes shows a cutaneous horn that is higher than the diameter of the base. Thus, clinical finding of erythema at the base of the cutaneous horn would be a reliable feature for early detection of squamous cell carcinoma.
\end{abstract}

Key words cutaneous horn; histopathology; squamous cell carcinoma

Cutaneous horn is a clinical term for a circumscribed, conical, markedly hyperkeratotic lesion in which the height of the keratotic mass is at least half of its largest diameter. The term refers to a reaction pattern and not to a specific lesion. However, various types of associated lesions such as squamous cell carcinoma (SCC) may be found at the base of a cutaneous horn. Here, we report a case of SCC manifested as a cutaneous horn and discuss a key to early detection of SCC with a cutaneous horn appearance.

\footnotetext{
Corresponding author: Kazunari Sugita, MD, PhD

sugita@med.tottori-u.ac.jp

Received 2018 February 19

Accepted 2018 March 11

Abbreviation: SCC, suquamous cell carcinoma
}

\section{PATIENT REPORT}

A 92-year-old man presented with a 3-month history of a slowly growing asymptomatic lesion on the left side of his neck. Physical examination revealed a keratotic cutaneous horn, $10 \mathrm{~mm}$ in height, appearing as a curved yellowish-white excrescence with a firm consistency on the left laterocervical region (Fig. 1a). At the base, there was an indurated erythema of $3 \mathrm{~mm}$ in diameter (Fig. 1a). He had no regional lymphadenopathy or other symptoms such as pain and pus discharge. The lesion was completely excised surgically. Histopathological examination revealed a hyperkeratotic horn developing over a mass of large atypical keratinocytes with large hyperchromatic and pleomorphic nuclei. The mass showed central crateriform invagination filled with parakeratotic keratinization forming a columnar keratotic horn between two hair follicles. The lesion had invaded into the deep dermis in association with lymphocytic infiltration (Figs. $1 \mathrm{~b}$ and c). On the basis of clinical and histopathological findings, a diagnosis of invasive cutaneous SCC was made.

\section{DISCUSSION}

In general, differential diagnosis for cutaneous horns includes filiform verruca, seborrheic keratosis, SCC, actinic keratosis, keratoachanthoma, Bowen's disease, trichilemmoma and basal cell carcinoma. ${ }^{1}$ However, most cutaneous horns are benign as was shown in a large study of 643 cases, of which $38.9 \%$ were malignant and $61.1 \%$ were benign lesions. ${ }^{2}$ Recent studies have also shown that the incidence of a benign histopathological base ranges from $41 \%$ to $60 \%$ and is up to $77 \%$ in cases of eyelid lesions. ${ }^{2,3}$

Actinic keratosis having the potential for progression to invasive SCC is the most common entity for the horn base. ${ }^{4} \mathrm{~A}$ recent study showed that cutaneous horns in association with invasive SCC had a tendency to have less height than the diameter of the base. ${ }^{5}$ In our case, however, the height of the cutaneous horn $(10 \mathrm{~mm})$ was larger than the diameter of the base $(3 \mathrm{~mm})$, indicating that invasive SCC sometimes shows a cutaneous horn that is higher than the diameter of the base. It is reported that the incidence of erythema at the base is higher for invasive SCC than for other entities including benign 


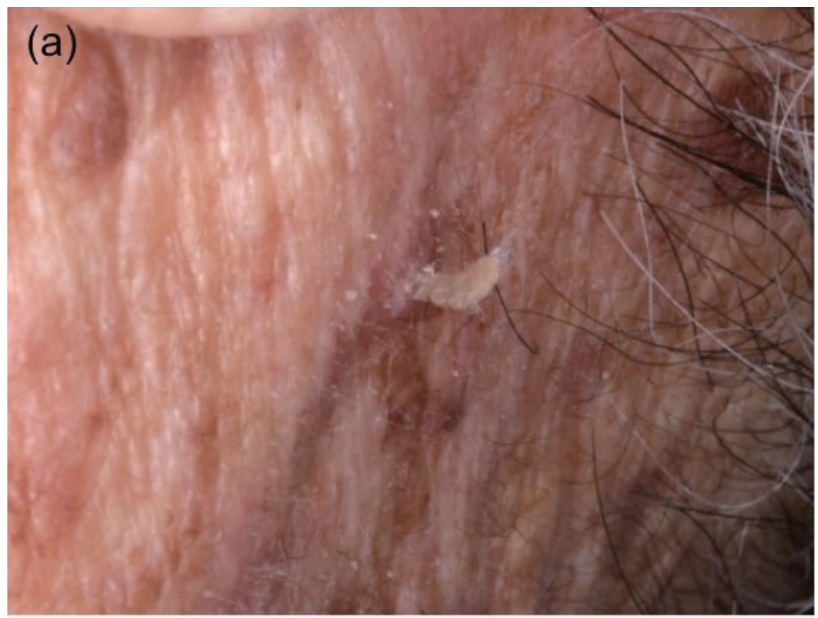

\section{(b)}

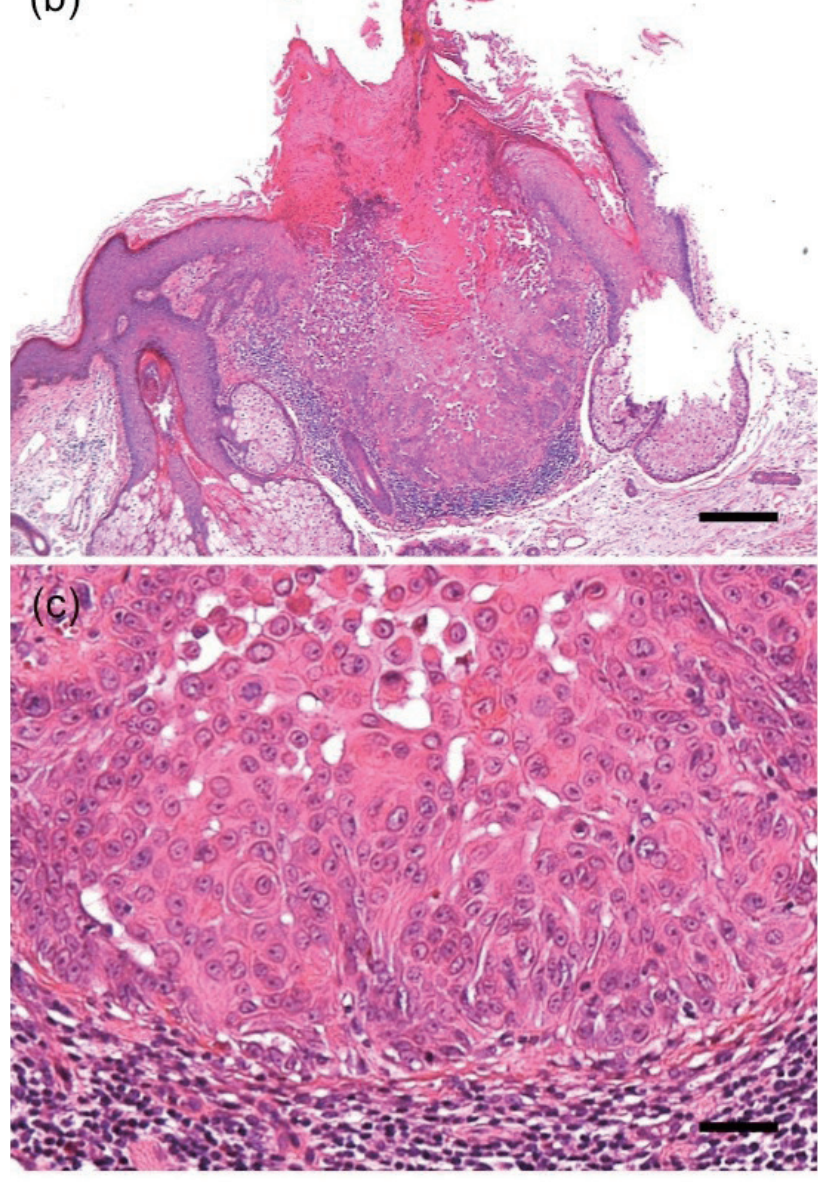

Fig. 1. (a) A keratotic cutaneous horn on his left side of the neck. (b) Lower magnification histopathology of the resected specimen (hematoxylin and eosin). Bar $=1 \mathrm{~mm}$. (c) A large well-differentiated keratinizing atypical cells in the dermis (hematoxylin and eosin). Bar $=25 \mu \mathrm{m}$. keratosis and actinic keratosis. ${ }^{5}$ Thus, our clinical finding of erythema at the base of the cutaneous horn supports the results of that study and it would be a reliable feature for early detection of SCC.

The authors declare no conflict of interest.

\section{REFERENCES}

1 Vano-Galvan S, Sanchez-Olaso A. Images in clinical medicine. Squamous-cell carcinoma manifesting as a cutaneous horn. N Engl J Med. 2008;359:e10. PMID: 18753642.

2 Yu RC, Pryce DW, Macfarlane AW, Stewart TW. A histopathological study of 643 cutaneous horns. Br J Dermatol. 1991;124:449-52. PMID: 2039721.

3 Mencía-Gutiérrez E, Gutiérrez-Díaz E, Redondo-Marcos I, Ricoy JR, García-Torre JP. Cutaneous horns of the eyelid: a clinicopathological study of 48 cases. J Cutan Pathol. 2004;31:539-43. PMID: 15268708.

4 Park H, Kim W, Kim H, Yeo H. Cutaneous Horn in Premalignant and Malignant Conditions. Arch Craniofac Surg. 2016;17:25-7. PMID: 28913248.

5 Pyne J, Sapkota D, Wong JC. Cutaneous horns: clues to invasive squamous cell carcinoma being present in the horn base. Dermatol Pract Concept. 2013;3:3-7. PMID: 23785640. 